\title{
Vertextungsstrategien in brasilianischen und deutschen Magistereinleitungen - eine exemplarische Analyse
}

\author{
Kathrin Schweiger ${ }^{1}$
}

\begin{abstract}
This article focuses on the rhetorical structure of introductory sections in Brazilian and German MA dissertations from the field of linguistics. The analysis is based on the CARS ("Create a Research Space") model proposed by Swales (1990) for examining introductions in English research papers. We also resort to Hutz (1997), who applies Swales' CARS model and compares English and German research papers from the discipline of psychology, and compare Brazilian with German text production (case study). The aim of this paper is to investigate whether Brazilian and German MA dissertations follow the same schematic patterns. Do the differences hold across the two cultures and different disciplines? To what extend can we talk about a 'universal academic discourse', as Widdowson (1979) claims?
\end{abstract}

Keywords: Contrastive genre analysis in academic settings; scientific language; academic introductions.

Resumo: O artigo analisa a estrutura retórica da "introdução" em dissertações brasileiras e alemãs da área de lingüística (estudo de caso). Tomando como base o modelo CARS ("Create a Research Space") de Swales (1990) e Hutz (1997) sobre a estrutura genérica de introduções de artigos acadêmicos em inglês, o objetivo desta pesquisa é investigar se as introduções de dissertações seguem um esquema comunicativo-proposicional, e, se for o caso, de que maneira se distinguem as introduções em relação às culturas e disciplinas. Até que ponto se pode falar da "universalidade do discurso acadêmico", como proclama Widdowson (1979)?

Palavras-chave: Gêneros textuais acadêmicos; Gêneros textuais acadêmicos em contraste; Linguagem científica; Introduções acadêmicas.

\section{O. Einleitung}

Auf einem internationalen Kongress des DAAD (Deutscher Akademischer Austauschdienst) in São Paulo zum Thema „Wissenschaftlerbiografien, Wissenschaftlermobilität, Wissenschaftlermigration“ im Juni 2007 wurden die Auswirkungen der Globalisierung auf den Wissenschaftsbetrieb diskutiert und erörtert. Auch die Universitätssysteme müssen sich dem „Druck der Globalisierung“ stellen. In Europa zeigt sich dies am „Bologna-Prozess“, bei dem sich 1999 die europäischen Universitäten auf ein einheitliches Studiensystem einigten. Bis zum Jahr 2011 soll daher an allen Universitäten das Bachelor- und Mastersystem eingeführt sein. Internationale akademische Erfahrung spielt in der heutigen wissenschaftlichen Ausbildung eine

\footnotetext{
${ }^{1}$ Doutoranda do Programa de Pós-graduação em Língua e Literatura Alemã da Universidade de São Paulo.-schweiger@usp.br
} 
immer größere Rolle; Praktikums-Studien- oder Forschungsaufenthalte im Ausland werden als karriereförderlich angesehen. Die international mobile Studentenzahl wächst stetig an und nach Einschätzung des DAAD soll bis zum Jahre 2025 die 7-MillionenGrenze $^{2}$ überschritten $\operatorname{sein}^{3}$. Das Studium bzw. die wissenschaftlichen Institutionen müssen auf derartige berufliche sowie die Ausbildungssituation betreffende Konstellationen und internationale Karrieren vorbereitet sein. Vielfach werden daher internationale Kooperationen abgeschlossen bzw. Universitätspartnerschaften eingegangen, um einem gelingenden Auslandsaufenthalt die idealen Rahmenbedingungen zu geben. Mittlerweile studieren jährlich ca. $700^{4}$ deutsche Studenten an einer brasilianischen Universität.

Ein Studienaufenthalt in Brasilien verlangt neben der Aneignung des Portugiesischen - einer im deutschen Schulsystem nur sehr marginal angebotenen Fremdsprache - auch das Aneignen einer fremden Wissenschaftskultur. Dazu gehören in erster Linie die universitären Text $^{5}$ - und Diskursarten ${ }^{6}$ wie z.B. - die im deutschsprachigen Raum vorherrschende - Seminararbeit, das Referat, das Protokoll etc. und auch die Magisterarbeit, die dissertação de mestrado, die Gegenstand des vorliegenden Artikels sein soll. Gerade die Analyse und die darauffolgende Deskription akademischer Textarten ${ }^{7}$ und ihrer inhärenten Wissenschaftssprachen kann zu einem

\footnotetext{
${ }^{2}$ Die Zahl geht auf Berechnungen des australischen Bildungsinstituts IDP und des Konsortiums GATEGermany zurück (DAAD-Marketing-Büro, São Paulo vom 15.04.08).

3 Migration war allerdings immer schon Bestandteil einer wissenschaftlichen Laufbahn. Schon Wissenschaftler im Mittelalter zeichneten sich durch eine herausragende Mobilität aus. Ihr Anteil an der mobilen Bevölkerung war beträchtlich (EHLICH 2001). Auch die einsetzende Nationalisierung der Wissenschaften ab dem 16. Jahrhundert tat dieser Entwicklung keinen Abbruch. Die Motive für die „Wanderbewegungen“ der Wissenschaftler änderten sich allerdings mit Beginn des 20. Jahrhunderts. Vordergründig ging es nicht mehr um den wissenschaftlichen Austauschgedanken, sondern der Überlebensgedanke wurde - während der nationalsozialistischen Diktatur - zentral für die Emigration deutscher Wissenschaftler. Heute sind es vor allem auch ökonomische Anreize, die zu einem Anstieg der internationalen Mobilität führen (ebd.).

4 Daten von der DAAD-Außenstelle, 21.11.2007 in einem Interview des Deutschlandfunks (http://www.dradio.de/dlf/sendungen/campus/699673/; Zugang 20.06.08).

5 „Eine Textart ist eine zweckbezogene Tiefenstruktur, die spezifische kommunikative Aufgaben zu bearbeiten gestattet, denen repetitiv immer neue Handelnde gegenüberstehen. Textarten sind also Großmuster des sprachlichen Handelns, denen eben jener Ressourcencharakter zukommt, der für die Bearbeitung gesellschaftlicher Zwecke unumgänglich ist“" (EHLICH 2000:11). Zentral für Ehlichs Textdefinition ist der gesellschaftliche Zweck, das heißt, dass der spezifische Zweck einer Textart X in einen größeren gesellschaftlichen Zusammenhang eingebunden ist - hier in die Institution der Wissenschaft; der Universität. Institutionen spiegeln Textarten in größerer Form wider, indem sie ebenfalls „Strukturen repetitiver Handlungen“ (EHLICH/REHBEIN 1994: 317) darstellen.

${ }^{6}$ Zur Unterscheidung zwischen Text und Diskurs basierend auf der Überlieferungsqualität von Texten s. EHLICH (1983).

${ }^{7}$ Es wird hier nicht der in der deutschen Textlinguistik häufig verwendete Begriff der „Textsorte“ verwendet, sondern „Textart“, der nach EHLICH (1990), etymologisch begründet, treffender erscheint. Im englischsprachigen Raum spricht man in fachsprachlicher Hinsicht von „genre“ bzw. von der „Genre
} 
erfolgreichen Auslandsstudium verhelfen, indem schon die Heimatuniversitäten ihren rein sprachpraktischen Unterricht um Vorbereitungskurse zum Wissenschaftsportugiesisch/akademischen Portugiesisch ${ }^{8}$, Französisch, Italienisch usw. beziehungsweise zum Wissenschaftsdeutsch erweitern.

Wissenschaft ist immer noch - so der allgemeine Tenor - „(...) trotz Berufung auf universale Vernunft in die geschichtliche Überlieferung (...) einer Gesellschaft eingebunden (...)“ (REUTER 2003: 324) und tief mit den Normen und Werten dieser verwurzelt ${ }^{9}$. Daher kann es immer wieder vorkommen, dass eine studentische wissenschaftliche Arbeit aus einem wissenschafts(sprachlichen) Kulturkreis als Textart als solche akzeptiert wird und in einem anderen Wissenschaftskulturkreis als inakzeptabel eingestuft wird. Textarten, denen der gleiche Zweck zugrunde liegt, die aber aus unterschiedlichen Kulturkreisen kommen, weisen nämlich Unterschiede in ihren Textmustern auf ${ }^{10}$. Daher besteht die zentrale Fragestellung der Arbeit in der Ermittlung gemeinsamer und/oder unterschiedlicher Vertextungsmuster brasilianischer und deutscher Studenten beim Verfassen der Textart Magisterarbeit. Aufgrund des Umfangs der Magisterarbeiten zeigte sich allerdings sehr bald, dass eine Gesamttextanalyse, das heißt die totale Erfassung der Magisterarbeit aus zeittechnischen, methodischen und finanziellen Mitteln schwer bis unmöglich ist, sodass eine Fokussierung auf einen Teilaspekt der Magisterarbeit vorgenommen werden musste. Als wichtigen, wenn nicht zentralen Teiltext ${ }^{11}$, wurde daher die „Einleitung“ als Untersuchungsgegenstand herangezogen. Ihr fällt bei der Auswahl bzw. Bewertung eines Textes eine Schlüsselfunktion zu (GnUtzMANN 1992). Bekanntlich sollen eine gute Einleitung und ein guter Schluss die Lektüre einer Arbeit „überflüssig“ machen.

\footnotetext{
Analysis", die vor allem von Swales (1990) und Bhatia (1993) geprägt worden ist und so auch in der brasilianischen Forschung unter der Bezeichnung Análise de gênero textual Einzug gefunden hat.

${ }^{8}$ Was zum Teil auch schon geschieht. Meines Wissens werden an der Technischen Universität in Berlin Kurse zu „Portugiesisch als universitäre Sprache“ angeboten (www.zems.tu-berlin.de; Zugang 20.6.08) sowie an der FU Berlin. Beispielhaft sei hier auch die Ludwig-Maximilians-Universität München genannt, die unter dem Namen „kommUNIkation“ ein Projekt implementierte, mit der Zielsetzung ihre Fach- bzw. Fremdsprachendozenten in der Vermittlung spezieller Fertigkeiten, die das universitäre Umfeld verlangt, fortzubilden. An der USP und PUC São Paulo existieren ebenfalls schon Kurse zum wissenschaftlichen Arbeiten für ausländische Studenten.

${ }^{9}$ Dies gilt besonders für die Sozial-und Geisteswissenschaften, die nach GNUTZMAnN (1992) vielmehr nationalgeprägt sind als beispielsweise die Naturwissenschaft, die einen „kulturabhängigen bzw. kulturübergreifenden Charakter hat"“ (a.a.O., 268).

${ }^{10}$ Zum kulturkontrastiven Vergleich studentischer Seminararbeiten s. KAISER (2002) sowie EßER (1997). Erstere zu venezolanischen Arbeiten letztere zu mexikanischen Arbeiten.

${ }^{11}$ Die „Einleitung“ verstehe ich in Anlehnung an OLDENBURG (1992:63) als Teiltext. „Teiltexte sind relativ abgeschlossene Einheiten, die gemeinsam im Zusammenwirken den Text konstituieren und in den Textzusammenhang eingebettet sind“(ebd.).
} 
Im Rahmen einer exemplarischen Analyse werden zwei Magistereinleitungen aus einem bisherigen Korpus von 25 deutschen sowie 25 brasilianischen Arbeiten der Disziplin Linguistik verglichen. Die brasilianische Arbeit stammt aus der Fakultät für Literatur- und Sprachwissenschaften der Germanistik, Universität São Paulo und umfasst 522 Wörter. Die deutsche Arbeit wurde am Institut für Deutsch-alsFremdsprache/Transnationale Germanistik, München, verfasst und umfasst 639 Wörter. Schwerpunkt der Analyse bildet der vergleichende Textaufbau. Als Grundlage der Analyse wird das Textaufbaumodell für „Einleitungen“ wissenschaftlicher Artikel von SwALES (1990) respektive HuTZ (1997) herangezogen und im folgenden Kapitel vorgestellt.

\section{Textaufbaumodelle für die Teiltextart „Einleitung“}

SWALES entwickelte $(1981,1990)$ als einer der Ersten ein inhaltlich-funktionales Modell (CARS-Modell: „Create a Research Space“), um Aufbau und Struktur von Artikeleinleitungen zu erfassen. Er geht von einem Stufenmodell aus, das in so genannte moves und steps unterteilt ist. Unter moves werden die größeren Teiltexteinheiten verstanden, während die steps als Untereinheiten zu verstehen sind.

Abb. 1

Move 1: Establishing the field

Step 1: Claiming centrality

Step 2: Making topic generalization(s) and/or

Step 3: Reviewing items of previous research

\section{Move 2: Establishing a niche}

Step 1A: Counter-claiming or

Step 2A: Indicating a gap or

Step 1C: Question raising or

Step 1D: Continuing a tradition 


\section{Move 3: Occupying the niche}

Step 1A: Outlining the niche

Step 1B: Announcing present research or

Step 2: Announcing principal findings

Step 3: Indicating research article structure

Es folgten eine Reihe kontrastiver Untersuchungen ${ }^{12}$, die sich das SwalesModell als Vorbild genommen haben. Allerdings gibt es nur einen Beitrag, der sich mit Einleitungen zu Magisterarbeiten beschäftigt. DudLEy-Evans (1986) stellt fest, dass Swales' CARS-Modell nicht eins zu eins auf MSc.-Arbeiten zu übertragen ist - ihnen liegt ein anderer Aufbau zugrunde, sodass er es um drei Teiltexteinheiten erweiterte. Nach meinen bisherigen Untersuchungen zeigte sich aber, dass sich das Modell von DUDLEY-EVANS (1986) auch nicht über deutsche und brasilianische Magisterarbeiten der Linguistik „stülpen“ lässt. Die Untersuchungen DuDLEY-EVANS beziehen sich auf englische Arbeiten des Faches Biologie - hier scheint ein anderes Textmuster vorzuliegen. Als Grundlage der vorliegenden Analyse dient das von GNutzMAnN/LANGE (1990) und von Hutz (1997) modifizierte bzw. reduzierte SwalesModell, das für die Untersuchung geeigneter erscheint, obgleich es sich auf die Textart „wissenschaftlicher Artikel“ bezieht. Es handelt sich bei einer Magisterarbeit allerdings um ein Großformat des wissenschaftlichen Artikels, sodass davon auszugehen ist, dass beide Textarten ein gros an Ähnlichkeiten aufweisen. Im Folgenden wird das abgewandelte CARS-Modell von HUTZ (1997: 117) vorgestellt:

\section{Einführung in den Untersuchungsgegenstand (1) (mit folgenden Varianten) Bedeutung des Untersuchungsgegenstandes (1A)}

\footnotetext{
${ }^{12}$ Beispielhaft seien hier aufgeführt: GNUTZMANN/OLDENBURG (1991): deutsch-englische Artikel der Linguistik, TAYLOR/CHEN (1991): chinesisch-englische Artikel der „hard science“; MEURER (1997): brasilianische Artikel; GOLEBIOWSKI (1999): polnisch-englische Artikel der Psychologie; ARVAY/TANKÓ (2004) ungarisch-englische Artikel der Linguistik.
} 
In diesem ersten Teiltextsegment rührt der Autor sozusagen die Werbetrommel für seine Arbeit. Hier geht es darum, den Leser von der zentralen Stellung und der Aktualität des Themas zu überzeugen. ${ }^{13}$

Beispiele:

Das Thema Interkulturalität gewinnt seit der steigenden weltweiten wirtschaftspolitischen Zusammenarbeit, dem Zusammenschluss der Europäischen Union und dem Schengener Abkommen zu Beginn des Jahres 1995 für jeden einzelnen von uns an Bedeutung (D14) ${ }^{14}$.

[Hervorhebung K.S.]

Nas últimas décadas, principalmente na América do Norte, uma área vem ganhando bastante espaço (...), o estudo das estratégias de aprendizagem (B 10).

[Hervorhebung K.S.]

Zentrale Ausdrücke entstammen aus dem Symbolfeld ${ }^{15}$ „Interesse“, „Bedeutung“, „Zentrum“ und ihren adjektivischen/adverbialen Ableitungen. Auch temporaldeiktische Ausdrücke (in Form einer Präpositionalphrase) sind häufig anzutreffen (in den letzten Jahren, in letzter Zeit).

\section{Allgemeine Aussagen zum Untersuchungsgegenstand (1B)}

Als weitere Möglichkeit der Ein- bzw. Hinführung zum Thema werden bisherige, allgemein bekannte Ergebnisse und Erkenntnisse des Forschungsfeldes aufgeführt. Das kann nach HuTz (1997) auch eine Definition sein oder das Angeben von allgemeinen Charakteristika des jeweiligen Untersuchungsgegenstandes bzw. Themas.

\section{Beispiel:}

Digitale Ethnographie ist eine Methode der Kulturforschung, die in den USA zur Erforschung von Denkhaltungen und Lerngewohnheiten bei Kindern entwickelt wurde. Dabei verbinden sich klassische ethnografische Aufzeichnungs- und

\footnotetext{
${ }^{13}$ SwALES (1991: 144) spricht hier von den centrality claims. "It seems to me that the way in which academics typically attract readerships is by an initial move which indicates that they are working in a lively or significant research area, even if the appearance of their title might indicate the contrary" (SWALES 1981: 23).

${ }^{14}$ Die Siglen Dx bzw. Bx stehen für ,deutsche Einleitung“/,brasilianische Einleitung“ sowie deren Nummerierung im Korpus.

${ }^{15}$ Symbolfeldausdrücke konstituieren sich aus Adjektiven, Adverbien Nomina und Verben und haben nach K. BüHLERS Sprachtheorie (1934) eine nennende Funktion im Gegensatz zu den situationsgebundenen deiktischen Ausdrücken (s. EHLICH 1996).
} 
Interpretationsmethoden mit der modernen Kommunikationstechnologie zur digitalen Ethnographie (D24).

\section{Literaturbericht (2)}

In diesem Teiltextsegment geht es um die Aufzählung bzw. das Wiedergeben des bisherigen Forschungsstandes - dabei kann der Literaturbericht aus einer erschöpfenden Auflistung von Literaturangaben bestehen und/oder nach GNUTZMANN/LANGE (1997, 96) mehr oder weniger umfassende inhaltliche Erläuterungen zu den einzelnen referierten Arbeiten geben. Diese Literaturlage kann auch als eine „pauschale Charakterisierung“ (ebd.) mit dem Verweis auf eine einzelne Arbeit aufgeführt werden. Die Berufung auf vorhergegangene Forschungsergebnisse kann zugleich als eigene methodisch/theoretische Grundlage dienen.

\section{Beispiele:}

In der gegenwärtigen Diskussion konkurrieren verschiedene linguistische Bezeichnungen für die Diskurspartikeln, z.B. „Abtönungspartikeln“ (Weydt 1969), „modale Partikeln“ (Krivonosov 1977) und „Sprechhandlungsaugmente“ (Rehbein 1979) (D 13).

Autores como Solmecke (1997) consideram a compreensão auditiva como parte de incompetência de entendimento mais abrangente e, portanto, como um caminho importante para o domínio de uma língua estrangeira (...) (B 10).

Das Teiltextsegment „Literaturbericht” befindet sich im Gegensatz zu Swales CARSModell bei Gnutzmann/LAnge (1990) und Hutz (1997) aus dem Teiltextsegment [1] herausgelöst.

\section{Legitimierung der Forschungsarbeit ${ }^{16}$ (3)}

In diesem Teiltextsegment manifestiert und legimitiert der Autor seine Arbeit, indem er auf Defizite und Probleme in der bisherigen Forschung hinweist und er durch seine Arbeit dementsprechend diese Lücke schließen möchte (counter-claiming). Die

\footnotetext{
${ }^{16}$ Nach SwALES (1990): Establishing a niche. HUTZ (1997) gibt für diesen inhaltlich-funktionalen Schritt zwei Varianten an: „3A Problematisierung von Forschungsergebnissen“ und „3B Aufzeigen von Forschungsdesiderata und Fragekomplexen“. Die Schritte sind schwer zu trennen (wie Hutz selbst sagt), sodass hier die beiden Varianten nur als ein Teiltextsegment gewertet wird.
} 
Bezugnahme auf die themenrelevante Literatur erfolgt daher oftmals in einer kritischen Art und Weise, indiziert durch adversative Ausdrücke des operativen Feldes ${ }^{17}$ wie allerdings, leider, jedoch, aber etc., Adverbien der Intensität (wenig, sehr, viel), Negationsausdrücke, Verben aus dem Handlungsbereich „kritisches Forschungshandeln“ sowie Substantive des entsprechenden Symbolfeldes (Defizit, Fehlen, Lücke usw.). Anstatt der rein defizitorientierten Vorgehensweise kann der Autor aber auch an vorhergehende Arbeiten, Traditionen anknüpfen, indem er zusätzlich neue Fragen aufwirft bzw. Forschungsdesiderata äußert, die natürlich auch an der unzureichenden Forschungslage liegen, indem bestimmte Aspekte eines Themas unzureichend bzw. noch nicht untersucht worden sind, dennoch scheint dieser Schritt weniger ,,aggressiv“ zu wirken.

\section{Beispiele:}

Nur wenige Forschungsarbeiten befassten sich bisher damit, welche Auswirkungen auf das Lernverhalten und auf den Lernerfolg die multimedialen Anwendungen tatsächlich haben (D23). [Hervorhebung K.S.]

Embora haja vários trabalhos sobre a entoação de algumas línguas como inglês (...), nota-se uma deficiência de pesquisa nesse sentido relacionadas ao português do Brasil (B4). [Hervorhebung K.S.]

\section{Vorstellung der eigenen Arbeit (4)}

\section{Zielsetzung der Arbeit (4A)}

Hier geht es um die Vorstellung der eigenen Arbeit bzw. die Nennung des eigentlichen Untersuchungsvorhabens (ocupying the niche).

\section{Beispiele:}

In dieser Arbeit geht es darum, sprachliche Verfahren im Original und der Übersetzung zu untersuchen, die die Rezeption des Lesers steuern (D17). [Hervorhebung K.S.]

Esta dissertação tem por objetivo primeiramente avaliar criticamente um modelo consensual (...) (B3). [Hervorhebung K.S.]

\footnotetext{
${ }^{17}$ Die Leistung des operativen Feldes besteht in der Verknüpfung bzw. in der Verarbeitung von Propositionen (s. EHLICH 1996).
} 


\section{Theoretische Grundlagen und Methodik der Arbeit (4B)}

Hier werden diejenigen Äußerungen des Autors subsumiert, die sich auf das methodische Vorgehen der Arbeit beziehen bzw. die theoretischen Grundlagen erörtern.

\section{Beispiele:}

Um die Darstellung der Kultur Puerto Ricos in der Literatur zu überprüfen, wird in der vorliegenden Arbeit eine Inhaltsanalyse der Tagespresse durchgeführt werden (D11).

Para chegar aos resultados (...) foi necessária a análise de um corpus de língua escríta, para verificar o uso real do modo subjuntivo em ambos os idiomas (B11). [Hervorhebung K.S.]

\section{Hypothesen (4C)}

Die Vorstellung der eigenen Arbeit bzw. deren Zielsetzung kann auch in der Aufstellung von Hypothesen und deren Überprüfung erfolgen. Dieses Teilsegment wird häufig mittels operativer Prozeduren (kausale und konsekutive Konnektoren) wie z.B. folglich, daher oder entsprechend eingeleitet, die die inhaltliche Verankerung zu den vorangegangenen Teiltextsegmenten anzeigen, da sich die Hypothesenbildung oftmals an die vorausgegangene theoretische Diskussion anschließt. Es ist davon auszugehen, dass das Vorkommen dieses Teilsegmentes in Magisterarbeiten geringer Art ist. Die mentale Tätigkeit „Hypothesen bilden“ setzt breite und vertiefte Kenntnisse eines Wissensbereiches voraus. Laut Studienordnung (s. USP-Studienordnung) fällt diese intellektuelle Tätigkeit noch nicht unter das Anforderungsprofil eines noch in der Novizensituation befindlichen Magistranden. Bei der Ausarbeitung einer Magisterarbeit geht es vielmehr um das Überprüfen vorhandener Theorien unter Anwendung der wissenschaftlichen Methodik sowie um das Sichten und das systematische Zusammenfassen der gegenwärtigen Forschungsliteratur.

\section{Beispiel:}

Minha hipótese de trabalho, portanto, deriva da observação práctica do processo de tradução (...) pode ser assim definida: (...) (B3). 


\section{Hinweise auf die Textstruktur (4D)}

Der Autor gibt in diesem Teil der Arbeit explizite Hinweise über die Textstruktur, um dem Leser zum einen eine Orientierungshilfe an die Hand zu geben und zum anderen dessen Leseerwartung zu wecken. HuTz $(1997,123)$ geht davon aus, dass dieses Teiltextsegment in seiner untersuchten Textart „wissenschaftlicher Artikel“ selten vorkommt; wenn man die relative Kürze wissenschaftlicher Artikel in Betracht zieht und deren übersichtlich gegliederte Überschriften. In den Magisterarbeiten ist dieses Teiltextsegment essenzieller Bestandteil der Arbeiten und im untersuchten Korpus auch immer belegt worden.

Beispiele:

Die ersten drei Kapitel stellen die theoretische Fundierung dar: Nach einer Erläuterung der Grundbegriffe (Kapitel 2) und einer Darstellung des Forschungsstandes der Interkulturellen Didaktik (3. Kapitel) werden verschiedene Dimensionen von Interkulturalität im FUS knapp skizziert (4. Kapitel) (D 14).

Iniciamos e estudo apresentado, no item II. EMBASAMENTO TEÒRICO (B 10).

\section{Gegenüberstellung deutsche versus brasilianische Einleitung}

Abb. 2

\begin{tabular}{|l|l|l|l|}
\hline 1A & $\begin{array}{l}\text { 0. Einleitung [D5] } \\
\text { Über die Bedeutung }\end{array}$ \\
$\begin{array}{l}\text { von grammatischen Regeln haben } \\
\text { verschiedene Richtungen rer der } \\
\text { fremdsprachendidaktischen Forschung } \\
\text { sehr unterschiedliche (...) Empfehlungen } \\
\text { gegeben. }\end{array}$
\end{tabular} \mid $\begin{aligned} & \text { 4A } \\
& \text { 1B }\end{aligned}$\begin{tabular}{l}
$\begin{array}{l}\text { Ein Sprachunterricht, der einerseits } \\
\text { einfach Grammatikunterricht ist und } \\
\text { grammatische Strukturen anbietet, ist } \\
\text { nicht ausreichend (...) } \\
\text { Andererseits (...) }\end{array}$ \\
$\begin{array}{l}\text { No presente trabalho tratarei do } \\
\text { emprego do modo subjuntivo em } \\
\text { alemão e em português, um estudo } \\
\text { teórico sobre o assunto, e depois a } \\
\text { análise de um corpus bilíngüe alemão- } \\
\text { português (..) }\end{array}$ \\
$\begin{array}{l}\text { A análise do corpus possibilitará o } \\
\text { levantamento e a comparacão dos } \\
\text { tipos de uso do subjuntivo nos dois } \\
\text { idiomas, a fim de estabelecer as } \\
\text { equivalências e as diferenças }\end{array}$ \\
\hline
\end{tabular}

\footnotetext{
${ }^{18}$ Die fettgedruckten Ausdrücke dienen als zentrale Indikatoren, die die Zuordnung zu einem Teiltextsegment erlauben.
} 


\begin{tabular}{|c|c|c|c|}
\hline & Gerade Lerner verfügen über (...) & & \begin{tabular}{|l} 
existentes. \\
\end{tabular} \\
\hline 3 & $\begin{array}{l}\text { Tatsache ist jedoch... } \\
\text { Oft bemängeln aber Lehrer und Lerner } \\
\text { das Fehlen von Übungen und beklagen } \\
\text { sich über die Reihenfolge und Art der } \\
\text { Präsentation von Regeln, die ihnen nicht } \\
\text { logisch erscheint. }\end{array}$ & 4D & $\begin{array}{l}\text { Num segundo momento, } \\
\text { concentrarei-me (...). } \\
\text { Descrevo abaixo o objetivo, a } \\
\text { justificativa e a metodologia. }\end{array}$ \\
\hline $4 \mathrm{~A}$ & 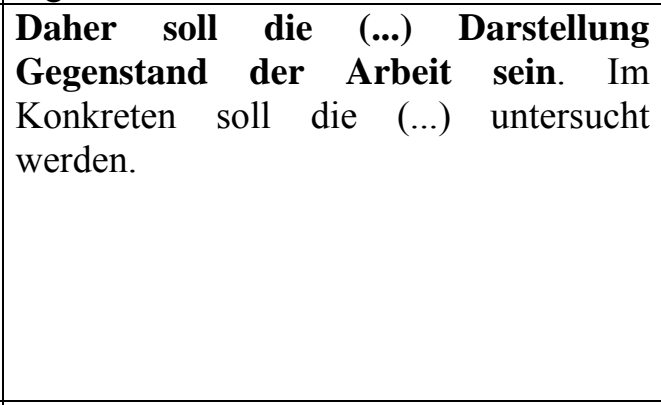 & $4 \mathrm{~A}$ & $\begin{array}{l}\text { 1.1. Objetivo e justificativa do } \\
\text { trabalho } \\
\text { Neste trabalho tenho como objetivo } \\
\text { a descrição e a comparação (...) a fim } \\
\text { de estabelecer as equivalências e as } \\
\text { diferenças entre os dois idiomas. Esta } \\
\text { compação poderá fornecer bases } \\
\text { para a elaboração de materiais (...) }\end{array}$ \\
\hline $4 \mathrm{~B}$ & $\begin{array}{l}\text { Für den Fremdsprachenunterricht ist es } \\
\text { laut Megen (1992,120) hierbei wichtig, } \\
\text { die Regularitäten der Pluralbildung so } \\
\text { darzustellen, dass sie den Sprachlernern } \\
\text { als „Regeln mit maximalem } \\
\text { Anwendungsbereich vermittelt werden } \\
\text { können“. }\end{array}$ & $4 \mathrm{~B}$ & $\begin{array}{l}\text { A princípio, meu objetivo era } \\
\text { descrever e comparar todos os tipos } \\
\text { (...). Então realizei um levantamento e } \\
\text { numa primeira comparação de (...). } \\
\text { Como não seria possível analisar com } \\
\text { mais profundidade, de (...) foi } \\
\text { necessário fazer um recorte (...) Por } \\
\text { fim, decidi apresentar um panorama } \\
\text { contrastivo (...), e me aprofundar mais } \\
\text { (...) para chegar à descrição dos fatores } \\
\text { (...). } \\
\text { A opção por esse recorte se justifica } \\
\text { tanto pela (...) quanto (...). }\end{array}$ \\
\hline $2 *$ & $\begin{array}{l}\text { Bei der Frage der Regelhaftigkeit } \\
\text { scheinen die Auffassungen zu } \\
\text { divergieren. Während Linguisten wie } \\
\text { Augst (1975) und Mugdan (1977) zu } \\
\text { dem Ergebnis kommen (...), bestreiten } \\
\text { vor allem Psycholinguisten (Wode 1988) } \\
\text { und Spracherwerbsforscher (z.B. } \\
\text { Köpcke) - dies wiederum. }\end{array}$ & 3 & $\begin{array}{l}\text { As obras didáticas do alemão não } \\
\text { enfocam o emprego do modo (...). } \\
\text { Geralmente os trabalhos se limitam a } \\
\text { tratar (...). Algumas gramáticas citam } \\
\text { (...), mas também não abordam (...). E } \\
\text { não há trabalhos contrastivos (...) que } \\
\text { poderiam fornecer. }\end{array}$ \\
\hline 4D & $\begin{array}{l}\text { Im theoretischen Teil der Arbeit soll } \\
(\ldots) \\
\text { Des Weiteren werden (...). } \\
\text { Im zweiten, empirischen Teil der Arbeit } \\
(\ldots)\end{array}$ & $4 \mathrm{~B}$ & $\begin{array}{l}\text { É importante a utilização de um } \\
\text { corpus num trabalho como esse (...) } \\
\text { Não é suficiente ter como base apenas } \\
\text { gramáticas (...), além de eles trazerem } \\
\text { muito pouco sobre esse tema, eles } \\
\text { prescrevam regras, que, muitas vezes, } \\
\text { estão distantes do uso real da língua. }\end{array}$ \\
\hline $4 \mathrm{~A}$ & $\begin{array}{l}\text { Weiterhin sollen folgende Fragen bei der } \\
\text { Analyse beantwortet werden: } \\
\text { - Wann wird (...) eingeführt? } \\
\ldots\end{array}$ & & \\
\hline $4 \mathrm{D}$ & $\begin{array}{l}\text { Im Anschluss werden (...) präsentiert } \\
\text { (...). Vor dem Hintergrund der (...) } \\
\text { werden die Ergebnisse im Schlussteil } \\
\text { zusammengefasst. Darüber hinaus soll }\end{array}$ & & \\
\hline
\end{tabular}


(...) aufgezeigt werden.

* hierbei handelt es sich um eine Fußnote

\section{Deskriptive Analyse der deutschen Einleitung}

In einem ersten Schritt erfolgt der Einstieg mittels des von SwALES (1990) so genannten centrality claim. Der Autor vermittelt dem Leser, dass es sich bei dem vorliegenden Untersuchungsgegenstand, hier der Bedeutung der Grammatikvermittlung, um ein immer noch ungeklärtes - zentrales - fremdsprachendidaktisches Forschungsfeld handelt, das wohl immer wieder Anlass zur Diskussion gibt [1A].

In einem zweiten Schritt werden die gegensätzlichen Positionen dazu dargestellt; es wird hier allgemein bekanntes Wissen wiedergegeben und dem Leser, als Erinnerung, die verschiedenen Ansätze aufgeführt [1B].

Von diesen allgemeinen Ausführungen über das Für und Wider der Grammatikvermittlung führt uns der Autor dann auf das Lehrbuch, das - allgemein bekannt (denn Tatsache) „(...) oft die einzige Quelle für Grammatikdarstellungen - und - übungen [ist] “. Es klingt aufgrund des hier negativ konnotierten Ausdrucks einzige schon die kritisch-skeptische Haltung des Autors hinsichtlich dieses Umstandes durch. Mittels des Verbes bemängeln (nach Grimm: vituperare, tadeln, fehler in etwas finden, für mangelhaft, schadhaft erklären) wird dann explizit ein Defizit angesprochen [3]. Genauer genommen werden hier die Lehrwerke bzw. Lehrwerkautoren kritisiert. Hieraus leitet sich dann die Legitimation der Arbeit ab, die dann in der Äußerung der Zielsetzung der Arbeit [4A] mündet, eingeleitet durch die operative Prozedur daher, die einen schlussfolgernden-kausalen Charakter hat: „Daher soll die inhaltliche und visuelle Darstellung von Grammatikregeln in Lehrwerken ${ }^{19}$ Gegenstand der vorliegenden Arbeit sein". Im Konkreten soll die Präsentation der Einheit 'Vermittlung der Nominalplurale' in den DaF-Lehrwerken des Deutschen untersucht werden “.

Bei 4A zeigt sich die typische Formulierung von Zielsetzung im Deutschen in Form des Modalverbs sollen + Passivkonstruktion im Zusammenspiel mit einem Verb

\footnotetext{
${ }^{19}$ Innerhalb des Schrittes 4A fügt der Autor eine Fußnote ein, bei der es sich um eine „Definitionsfußnote“ (BRAND 1998) handelt; der Autor erläutert hier den Unterschied zwischen Lehrwerk und Lehrbuch: „Das Lehrbuch ist streng genommen [Hervorhebung K.S:] nicht zu verwechseln mit dem Lehrwerk (...)“. Hier verwendet der Verfasser einen Heckenausdruck, bei dem es sich um ein hedging im prototypensemantischen Sinn handelt.
} 
aus dem nach Fandrych (2002: 20) benannten Bereich des „logischen und praktischen Forschungshandelns". Bei untersuchen, das der deutschen Gemeinsprache entspringt kann man die ursprüngliche Bedeutung noch gut erkennen. An der räumlichen Partikel unter zusammen mit suchen kann man herleiten, dass es um das „Suchen unter der Oberfläche geht“, also, es sich um ein „tiefgründiges Suchen“ im Sinne wissenschaftlicher Tätigkeit handelt.

Die Verwendung des Modalverbs sollen im Handlungszusammenhang Zielsetzung könnte auf den Fremdsprachenlerner befremdlich wirken. Im Fremdsprachenunterricht wird dem Lerner vermittelt, dass bei der Verwendung des Modalverbs sollen es sich nicht um das eigenbestimmte Handeln, sondern um ein fremdbestimmtes Handlungseinwirken handelt. Es ist nämlich bei sollen nicht bestimmt, ob zielausführender und zielbildender Aktant identisch ist (s. REDDER 1983, JOHNEN 2003). In wissenschaftssprachlicher Hinsicht wird hier aber ausschließlich das Handlungsziel des Autors verbalisiert, denn der Autor ist - zumindest vordergründig alleiniger „Herr seiner Gedanken“ und entscheidet über deren Materialisierung.

Sollen als sprachlicher Indikator für Zielsetzung geht allerdings über diese Funktion hinaus. In funktional-pragmatischer Hinsicht (s. REDDER/BRÜNNER 1983, EHLICH/REHBEIN 1972) verbalisiert sollen „(...) die mentale Vorgeschichte von Handlungen im Hinblick auf Ziele und Bedingungen dieses Handelns (...)" (GRAEFEN 2000a: 118). Dieser vorgeschaltete Prozess der Entscheidungsfindung wird durch sollen mitgeteilt (ebd.).

GRAEFEN (2000a) weist daraufhin, dass sich die Verwendung von sollen dennoch als widersprüchlich erweist - das Moment der Entscheidung ist mit dem Entschluss der Verschriftlichung schon gefallen, sodass der Planungsprozess seinen Abschluss gefunden hat. M. E. handelt es sich hier nicht um einen Widerspruch, denn dem Leser wird so ermöglicht, die Gedankengänge des Autors zu rekonstruieren und involviert ihn in diesen, obgleich für den Autor der Prozess abgeschlossen ist. Nach GRAEFEN (a.a.O. : 9) haftet dieser modalisierenden Art der Äußerung daher etwas Fiktives ${ }^{20}$ an. Sie vermutet, dass die Funktion (man könnte hier m. E. von einer Art Nebenfunktion sprechen) darin besteht, „(...) dem Leser - der die Lektüre jederzeit abbrechen oder umgestalten kann - einen Handlungsspielraum zu gewähren.“ Die Funktion von sollen hat einen weniger kompromittierenden Charakter, wenn man

\footnotetext{
${ }^{20}$ Der Ausdruck ,fiktiv“ erscheint mir m.E. unglücklich gewählt.
} 
bedenkt, dass der portugiesischsprachige Autor unmittelbar den Leser vor „,vollendete Tatsachen stellt“ (s. z.B. „Ziel dieser Arbeit ist der Vergleich zwischen (...)“).

Zutreffend ist daher die Unterscheidung von JOHNEN (2003) (in Anlehnung an REHBEIN (1977), WundeRLICH (1981), REDDER (1983)), der bei dem Gebrauch der Modalverben weiterhin zwischen dem Prozess der Zielbildung und der Ausbildung einer Absicht zur Handlung bzw. einer Absicht, die den Entschluss der Verwirklichung der Absicht darstellt, differenziert. Laut dieser Unterscheidung fällt die Verbalisierung mit sollen im Deutschen unter den Prozess der Zielbildung, während im Portugiesischen das Handlungsziel: Objetivo do trabalho (Zielsetzung) entschlusskräftigeren Charakter besitzt und vor allem Verben der Kategorie Absicht ${ }_{2}$ verwendet werden.

Typische Verben, die eine vollzogene Absichtsausbildung indizieren, sind laut JoHNEN (2003, 113) Verben wie beabsichtigen, vorhaben, sich vornehmen - Verben, die im deutschen wissenschaftssprachlichen Umfeld bei der Verbalisierung des Handlungsziels Zielsetzung - nicht anzutreffen sind. Im Portugiesischen zählen Verben wie intencionar und propor-se dazu, die typisch für Zielsetzung in den brasilianischen Magisterarbeiten sind.

Als Absicht 2 -Verb kategorisiert JoHNEN $(2003,113)$ u.a. optar, das in dem vorliegenden Korpus an Magistereinleitungen ebenfalls vorkommt. Im Deutschen wären die Äquivalente entschließen sowie entscheiden, Verben, die im wissenschaftssprachlichen Gebrauch unüblich sind. In den vorliegenden brasilianischen Magisterarbeiten tauchen folgende entschlussverwirklichende Verben auf:

(B2) “Com esta pesquisa pretendo (...)”. $\rightarrow$ zwischen Absichtsbildung und Planung

(B8) 'Disposta a dar um passo nesse sentido, propus-me a realizar a presente pesquisa (...)". $\rightarrow$ vollzogene Absichtsausbildung

(B5) "Este trabalho propõe-se ao estudo contrastivo (...)". $\rightarrow$ vollzogene Absichtsausbildung

(B9) “Ao decidir continuar (...) optei por me dedicar à (...)".

$\rightarrow$ Entscheidung zur Verwirklichung des Ziels [Absicht $\left.{ }_{2}\right]$ 
Es folgt ein kurzer Hinweis auf bisherige Forschung in Form einer - auf eine einzelne Arbeit verweisende - integrierten Zitierung [2]; zugleich macht der Autor damit eine wichtige Aussage zu seinem methodischen Vorgehen, indem er bei seinen Untersuchungen zur Darstellung der Pluralbildung in Lehrwerken von der von „Megen (1992) postulierten Maxime ausgeht, dass die Regeln mit maximalem Anwendungsbereich vermittelt werden sollten ", sodass man diesen inhaltlichfunktionalen Schritt [2] auch als Schritt [4B] bewerten kann. Es folgen in Form einer Fußnote weitere Erläuterungen über den Forschungsstand hinsichtlich der Frage der Systematizität der Pluralformen. Warum die weitere Bezugnahme auf vorangegangene Forschung in die Fußnote „verbannt“" wurde, kann nur spekuliert werden. Es ist anzunehmen, dass dieser Teil der Forschung - die Kontroverse nämlich, ob den Pluralformen Systematizität zugrunde liegt oder nicht - nicht die Wichtigkeit besitzt, um im Haupttext Erwähnung zu finden bzw. hat in diesem inhaltlichen Schritt, was die Darstellungsform von Regeln in Lehrwerken betrifft, keine Relevanz. Nicht zuletzt werden die Arbeiten von drei der genannten Autoren ausführlich in den Folgekapiteln der Arbeit dargestellt.

Es folgen die Ausführungen zum Aufbau der Arbeit mit temporal- und lokaldeiktischen Elementen (im theoretischen Teil A; des Weiteren; im zweiten, empirischen Teil B) [4D]. Das Aufstellen der Fragen (,Weiterhin sollen folgende Fragen beantwortet werden: Wann wird der Plural eingeführt, Wie ausführlich wird die Einheit präsentiert etc. “) werte ich als ,Zielsetzung“ [4A]. Daraufhin folgt nochmal das Aufzeigen der Organisationsstruktur [4D] des Artikels: „Im Anschluss werden die Ergebnisse einer Umfrage präsentiert; „(...) werden die Ergebnisse im Schlussteil zusammengefasst“. Darüber hinaus soll ein alternativer Vorschlag zur Pluraldarstellung aufgezeigt werden.

Die Arbeitsschritte werden dann in der sprachlichen Form des unmodalisierten Passivs angekündigt, wobei im letzten Satz wieder eine soll-Konstruktion auftaucht. Hier scheint das Modalverb sollen - neben der Verbalisierung der Zielsetzung - noch eine andere Funktion wahrzunehmen. Der Autor scheint hier vielmehr mittels sollen einen Versuch eines alternativen Vorschlags zur Pluraldarstellung zu unternehmen aufgrund der ,,vorsichtigen Formulierung“ zeigt der Autor, dass

a) seine Wissenserarbeitung noch nicht abgesichert ist (,notwendiger Relativierung“ nach GRAEFEN 2000b: 8), sei es hier durch mangelnde empirische Überprüfung, ob 
diese neue Darstellung von den Lernern besser übernommen wird als die vorhergegangenen bzw. er will in funktionalpragmatischer Hinsicht dem Leser Handlungsspielraum ermöglichen, indem es an ihm (dem Leser) liegt, ob er den Vorschlag annehmen will oder nicht.

und/oder

b) der Autor hat hinlängliche Sicherheit über den Sachverhalt (hier: die alternative Pluraldarstellung ist die bessere), aber aus wohlüberlegten strategischen Motiven wählt er eine abschwächende Abfassung um sich - der Hedging-Theorie ${ }^{21}$ entsprechend - vor eventueller Ablehnung seitens des Lesers zu schützen (,taktisch-vorsichtige Abschwächung“ nach GRAEFEN 2000b: 8).

Da es sich hier aber „nur“ um eine Magisterarbeit handelt, werte ich diese sollen-Konstruktion als „,notwendige Relativierung“.

\section{Deskriptive Analyse der brasilianischen Einleitung}

Die brasilianische Einleitung ist durch eine Zweiteilung gekennzeichnet, und zwar, in den Abschnitt „Einleitung“ sowie „Zielsetzung und Begründung der Arbeit“. Unter der Überschrift „Einleitung“ beginnt die Arbeit mit dem Teiltextsegment „Zielsetzung/Objetivo de trabalho“ [4A]: „No presente trabalho tratarei do emprego (...)“, dem Leser wird ohne Umschweife die Zielsetzung der Arbeit mitgeteilt. Innerhalb des Paragrafen bzw. desselbigen Satzes erklärt der Autor den Aufbau seiner Arbeit mittels der temporaldeiktischen ${ }^{22}$ Ausdrücke primeiramente und depois [4D].

\footnotetext{
${ }^{21}$ Bei den Heckenausdrücken (engl.: hedging $=$ Hecke) handelt es sich um Ausdrücke, die die Sprechereinstellung modifizieren, ohne dass dabei die Bewertungsinstanz (z.B. Sprecher) genannt wird. Nach Clemen $(1998,14)$ haben sie eine pragmatisch-kommunikative Funktion und ermöglichen dem Sprecher: ,seine Aussagen zu subjektiveren, seine Verantwortung für den Wahrheitsgehalt der Proposition zu relativieren, den Grad seiner Gewissheit oder seines Zweifels über die Geltung seiner Feststellung einzuschränken, absolute Aussagen zu vermeiden, Verantwortung für Äußerungsinhalte zu transferieren, persönliche Einstellung zu bekunden und Sachverhalte zu bewerten“.

${ }^{22}$ Bei den Ausdrücken primeiramente und depois handelt es sich nach Ehlich (1992) genau genommen um krypto-deiktkische Elemente. Um Vagheit, die durch deiktische Ausdrücke entstehen kann, zu vermeiden, werden vermeintliche Symbolfeldausdrücke benutzt - dennoch haben diese einen situativen Charakter.
} 
Daraufhin folgen Ausführungen über die methodologischen Vorteile einer Korpusanalyse [4B] und es erfolgt eine indirekte Zielsetzung, indem der Autor erklärt, was die Korpusanalyse ermöglicht, nämlich Äquivalenzen und Differenzen aufzuzeigen.

Es folgen weitere textorganisierende Schritte mittels des temporaldeiktischen Elements num segundo momento (...)“ und durch die Lokaldeixis abaixo. Die Autorin leitet damit zum zweiten Teil der Einleitung über "Descrevo abaixo o objetivo, a justificativa e a metodologia deste trabalho “.

In dem Unterkapitel 1.1. „Zielsetzung und Begründung der Arbeit“ folgt repetitiv wieder die explizite Zielsetzung der Arbeit( "Neste trabalho tenho como objetivo (...)“.). Im Anschluss "wagt” der Verfasser die Behauptung, dass "esta comparação poderá fornecer bases para a elaboração de materiais didáticos sobre esse tema e subsídios para os aprendizes de alemão como língua estrangeira"[4]. Aufgrund des Modalverbs poder in seiner Funktion des Aufzeigens einer Handlungsalternative (Redder 2001) in der Futurform und in Verbindung des performativen Verbs fornecer wird diese Handlungsalternative abgeschwächt in eine ,vorsichtige Situationseinschätzung“ (a.a.O.).

Es erfolgt eine längere metakommunikative Passage (13 Zeilen), indem der Verfasser sich zu seinem methodologischen Vorgehen äußert und dieses rechtfertigt [4B]:

“A principio meu objetivo era (...)”.

“Então realizei (...)”.

“Por fim me decidi apresentar (...)”.

Durch das absichtsvollzogene Verb decidir wird der Prozess der methodologischen Entscheidungsfindung abgeschlossen. Es folgt anschließend eine Begründung hinsichtlich der Auswahl des Korpus “A opção por esse recorte se justifica (...) “, die die metakommunikativen Ausführungen beendet.

Es folgt dann die Legitimierung der Arbeit [3] durch das Aufzeigen von Forschungsdefiziten:

“As obras didáticas não enfocam o emprego (...)“.

“(...) os trabalhos se limitam a tratar (...)”.

“Algumas gramáticas (...) citam, mas também não abordam (...)”. 
“E não há trabalhos (...)”.

Entgegen der allgemeinen Erwartung, dass brasilianische Wissenschaftler Kritik in einer zurückhaltenderen Form formulieren bzw. auf diesen funktionalen Schritt oftmals gänzlich verzichten - wie MEURER (1995) konstatiert, erfolgt hier in recht ausführlicher und direkter Weise das Aufzeigen von Forschungsdefiziten. Die kritische Auseinandersetzung wird dabei vor allem mit der Negationspartikel não realisiert (das Verb limitar in seiner Grundbedeutung ist negativ konnotiert und nicht auf die Verneinungspartikel angewiesen). Die von FANDRYCH (2002) und Hund (1999) für die deutsche Wissenschaftssprache festgestellte Affinität der Begriffsbildung aus dem räumlich-physischen Metaphernbereich zeigt sich auch bei den hier verwendeten portugiesischen Sprechhandlungsverben. Es handelt sich dabei um limitar - begrenzen, nicht weiter gehen; abordar - erläutern, behandeln sowie focar.

abordar 1. chegar á beira ou borda de. 5. Tratar de, versar (tema, assunto). 6. Estar borda com borda; encostar; limitar (Dicionário Aurélio 1999: 12).

focar 1 . ópt. formar, por um meio de um sistema óptico (...) de maneira que forme imagens nítidas. 2. Fig. Pôr um foco; fazer voltar a atenção, o estudo, para; salientar, evidenciar (...) (Dicionário Aurélio 1999: 792).

limitar [lat. limitare] Não ir além; não passar, contentar-se, restringir-se, cingir-se (Dicionário Aurélio 1999: 1032).

Im vorletzten Absatz leitet die Autorin wieder zu den theoretischen Grundlagen [4B] über, indem sie die der Alltagssprache entstammende Kollokation aus dem bewegungsmetaphorischen Bereich "para chegar aos resultados" benutzt.

Es folgen weitere Ausführungen über Sinn und Zweck der von ihr vorgenommenen Methode: "É importante a utilização de um corpus num trabalho como esse, para poder comparar". Die Autorin betont bzw. appelliert noch einmal an die Wichtigkeit der Korpusanalyse in Form von deontischen Hinweisen wie“ $E$ importante“; "não é suficiente“ und schließt damit ihre Einleitung ab.

\section{Ergebnisse}

Bei der Gegenüberstellung beider Einleitungen ist festzustellen, dass in der brasilianischen Einleitung weder ein Teiltextsegment [1] (Bedeutung des 
Untersuchungsgegenstandes sowie allgemeine Aussagen dazu) noch ein Teiltextsegment [2] (Literaturbericht) auszumachen sind. Vielmehr haben in der brasilianischen Arbeit die inhaltlich-funktionalen Schritte „Zielsetzung“ [4A] und „Darlegung des methodischen Vorgehens“ [4B] einen größeren Stellenwert. Wohingegen bei der deutschen Arbeit Teiltextsegment [1A+1B] erscheint und in einer Zielsetzung mit „Daher sollen $X Y$ untersucht werden“ mündet. Die Funktion des Teiltextsegments [1] dient dazu, den Leser in die Problemstellung einzuführen und die Begründung [3] zu liefern, die zur Herausbildung der Fragestellung führt - die Zielsetzung mittels sollen verdeutlicht zusätzlich die mentale Vorgeschichte und involviert den Leser in den wissenschaftlichen Erkenntnisprozess, der ab dem Teiltextsegment [1] beginnt. Somit sehe ich die Thesen des deutschstämmigen australischen Wissenschaftlers CLYNES (1993) teilweise widerlegt, der deutschen Texten eine starke inhaltsbezogene Ausrichtung zuschreibt, die zugleich den Leser außen vorlässt. Gerade die sollen-Konstruktion verdeutlichen das Gegenteil. CLYNES These der Leserunfreundlichkeit deutscher Texte ist in diesem Aspekt zu widersprechen.

Beide Einleitungen sind zudem „leserfreundlich“ gestaltet, indem ausreichend Gebrauch von textorganisierenden Elementen gemacht wird. Dabei können in beiden Texten sowohl temporal- als auch lokaldeiktische Mittel ausgemacht werden (s. Anhang).

Die brasilianische Einleitung beginnt mit einer „unabhängigen“ Zielsetzung ${ }^{23}$ scheinbar ungebunden - ohne Einbettung in einen Handlungszusammenhang -, der Leser wird so unmittelbar über die Ziele und Themen der Untersuchung informiert ohne sich „einlesen“ zu müssen. Die Frage, ob wir es hier mit einer leserfreundlicheren Einleitung zu tun haben, sei dahingestellt - zweifelsohne wird der Leser schneller über den Textinhalt informiert.

Hinsichtlich der Darstellungshaltung des Autors ist eine verstärkte Autorenpräsenz aufgrund des vermehrten Gebrauchs von Personaldeixis in der ersten Person Singular bzw. des Possessivpronomens in der ersten Person Singular

\footnotetext{
23 GNUTZMANN (1991) stellt dies in ähnlicher Weise in anglo-amerikanischen wissenschaftlichen Einleitungen fest.
} 
auszumachen. Die deutsche Arbeit hingegen ist durch passivische Formen gekennzeichnet $^{24}$.

Insgesamt zeigt sich bei der Gegenüberstellung, dass die brasilianische Arbeit den Primärtext, d.h. den rein propositionalen Wissenschaftstext häufiger durch metakommunikative Einschübe durchbricht - die z.T. einen narrativen Charakter haben. Kulturhistorische Gegebenheiten zeigen (wie z.B. die Unterbindung der Ausbreitung von sekulären Schriften durch die portugiesische Krone), dass sich in Brasilien eine stärker oralisierte Kultur- und Kommunikationsgemeinschaft herausgebildet hat, deren Charakteristika sich noch in den heutigen Text- und Diskursarten ihren Niederschlag finden.

Zusammenfassend ist festzustellen, dass sich die vorliegenden Einleitungen im Aufbau und der Wahl der sprachlichen Mittel unterscheiden. Widdowsons (1979) These von der Universalität und Einheitlichkeit des wissenschaftlichen Diskurses könnte hier widersprochen werden - dennoch ist diese Behauptung mit Vorsicht zu genießen, da hier nur eine exemplarische Studie vorliegt und eine quantitative Ausweitung der Studie zur Erhaltung von verlässlichen Daten noch aussteht.

Anhang : Verteilung temporal-und lokaldeiktischer Elemente der Textorganisation

\begin{tabular}{|l|c|c|}
\hline Brasilianische Arbeit & temporaldeiktisch & lokaldeiktisch \\
\hline no presente trabalho & $\mathrm{X}$ & \\
\hline Primeiramente & $\mathrm{X}$ & \\
\hline depois (Krypto-Deixis) & $\mathrm{X}$ & \\
\hline Num segundo momento & $\mathrm{X}$ & $\mathrm{x}$ \\
\hline Abaixo & $\mathrm{X}$ & $\mathrm{x}$ \\
\hline neste trabalho & $\mathrm{x}$ & \\
\hline a princípio & $\mathrm{X}$ & \\
\hline Então & $\mathrm{x}$ & $\mathbf{2}=\mathbf{1 0}$ \\
\hline por fim & $\mathbf{8}$ & \\
\hline Para chegar ao resultado & & \\
\hline GESAMT & & \\
\hline Deutsche Einleitung & & \\
\hline
\end{tabular}

\footnotetext{
${ }^{24}$ Mittlerweile zeigt sich aber eine zunehmende Tendenz in deutschen wissenschaftlichen Texten, auf das Ich-Verbot zu verzichten und auf die 1. Pers. Singular zurückzugreifen. Man könnte gegenteilig argumentieren, dass der Verfasser zu seinen „Propositionen stehen sollte“ und sich nicht hinter deagentivierenden Strukturen verstecken sollte.
} 


\begin{tabular}{|l|c|c|}
\hline der vorliegenden & & $\mathrm{x}$ \\
\hline im theoretischen Teil & & $\mathrm{x}$ \\
\hline des Weiteren & $\mathrm{X}$ & $\mathrm{x}$ \\
\hline im zweiten Teil & $\mathrm{X}$ & \\
\hline Weiterhin & $\mathrm{X}$ & $\mathrm{x}$ \\
\hline im Anschluss & & $\mathrm{x}$ \\
\hline vor dem Hintergrund & & $\mathbf{5 = 8}$ \\
\hline darüber hinaus & $\mathbf{3}$ & \\
\hline GESAMT & & \\
\hline Insgesamt & & \\
\hline
\end{tabular}

\section{Literaturverzeichnis}

Anett A./ TANKÓ G. A contrastive analysis of English and Hungarian theoretical research article introductions. In: International Review of Applied Linguistics in Language Teaching 42/1/2004, 71-200.

BRAND, K. Fußnoten und Anmerkungen als charakteristisches Element wissenschaftlicher Darstellungsformen. In: DANNEBERG, L./ NiedERHAUSER J.(Hrsg) Darstellungsformen der Wissenschaft im Kontrast. Aspekte der Methodik, Theorie und Empirie. Tübingen, Narr 1997, 213-240.

Clemen, G. Hecken in deutschen und englischen Texten der Wirtschaftskommunikation: Eine kontrastive Analyse. Siegen, Univ., Diss., 1998.

Clyne, M. Pragmatik, Textstruktur und kulturelle Werte. Eine interkulturelle Perspektive. In: H., SCHRÖDER (Hrsg.) Fachtextpragmatik, Tübingen, Narr 1993, 3-18.

Dudley-Evans, A. Genre Analysis: An investigation of the Introduction and Method Section of MSc Theses. Coulthard, M. (ED.) Talking about Text. Discourse Analysis, Monograph No. 13. English Language Research. University of Birmingham, 1986.

EHLICH, K./REHBEIN, J. Einige Interrelationen von Modalverben. In: WundERLICH, D. (Hrsg.) Linguistische Pragmatik, Frankfurt, Lang 1972, 318-340.

Ehlich, K./ SteEts A. et al. Schreiben für die Hochschule. Eine annotierte Bibliografie. Frankfurt a. M., Lang 2000.

EHLICH, K. Funktional-pragmatische Kommunikationsanalyse: Ziele und Verfahren. In: L. Hoffmann (Hrsg.) Sprachwissenschaft - ein Reader. Berlin/New York, de Gruyter 1996,183-201.

EHLICH, K. Textsorten - Überlegungen zur Praxis der Kategoriebildung in der Textlinguistik. In: MACKELDEY, R. (Hrsg.) Textsorten/Textmuster in der Sprech- und Schriftkommunikation. Leipzig, Wissenschaftliche Beiträge der Universität 1990, 17-30. 
EHLICH, K. Deutsche Wissenschaftskommunikation - eine Vergewisserung. In: Jahrbuch Deutsch als Fremdsprache 27. München, iudicium 2001, 191-192.

EHLICH, K./ REHBEIN, J. Prolegomena zur Untersuchung von Kommunikation in Institutionen. In: BrünNer, G. / GrAefEN G. (Hrsg.) Texte und Diskurse. Methoden und Forschungsergebnisse der Funktionalen Pragmatik, Opladen, Budrich 1994, 287-327.

EHLICH, K. Scientific texts and deictic structures. In: SteIn, D. (ed.) Cooperating with written texts. Berlin/New York, de Gruyter 1992, 201-229.

EHLICH, K. Text und sprachliches Handeln. Die Entstehung von Texten aus dem Bedürfnis nach Überlieferung. In: Assmann J. /C. HARDMEIER (Hrsg.) Schrift und Gedächtnis. Beiträge zur Archäologie der literarischen Kommunikation. München, Fink 1983, 2443.

Eßer, R. Etwas ist mir geheim geblieben am deutschen Referat. Kulturelle Geprägtheitwissenschaftlicher Textproduktion und ihre Konsequenzen für den universitären Unterricht von Deutsch als Fremdsprache. München, iudicium 1997.

FANDRYCH, C. Herausarbeiten vs. illustrate: Kontraste bei der Versprachlichung von Sprechhandlungen in der englischen und deutschen Wissenschaftssprache. In: EHLICH, K. (Hrsg.) Mehrsprachige Wissenschaft - europäische Perspektiven, 2002, herunterladbar unter http://www.euro-sprachenjahr.de/onlinepub.htm

FerreirA, A.B.H. Novo Aurélio Século XXI: o dicinonário da língua portuguesa. 3. ed. Rio de Janeiro, Nova Fronteira 1999.

Gnutzmann, C./OldenburG, H. Contrastive text linguistics in LSP-research: theoretical considerations and some preliminary findings. In: SCHRÖDER, H.(ed.) Subject-oriented texts: languages for special purposes and text theory. Berlin, de Gruyter 1991, 103-136.

Gnutzmann, C. Kontrastive Fachtextlinguistik als Projektaufgabe: Theoretische Fragen und praktische Antworten. In: BAUMAnN, K.-D./ KALVERKÄMPER, H. (HrSG.) Kontrastive Fachsprachenforschung.. Tübingen, Narr 1992, 266-275.

Gnutzmann, C. /LANG, R. Kontrastive Linguistik und Fachsprachenanalyse. In: GnUtzMAnN, C. (Hrsg.) Kontrastive Linguistik. Frankfurt, Lang 1990, 85-116.

GNUTZMANN, C. Sprachliche Indikatoren zur Explizierung von „Zielsetzungen“ im Englischen und Deutschen. In: Fachsprache/International Journal of LSP 01/1991, 10-14.

GolebiowsKi, Z. Application of Swales' Model in the anaylisis of research papers by polish authors. In: International Review of Applied Linguistics in Language Teaching 37/1999, 231-247.

GRAefEN, G. Textkommentierung in deutschen und englischen wissenschaftlichen Artikeln. In: Schlosser, H.- D. (Hrsg.) Sprache und Kultur. Frankfurt a.M., Lang 2000a, 113-124.

Graefen, G. "Hedging" als neue Kategorie? Ein Beitrag zur Diskussion. 2000b . http://www.daf.uni-muenchen.de/downloads/hedge.pdf. 
GRIMMSCHE WÖRTERBUCH Band 1, Spalten 1457 - 1461, http://germazope.unitrier.de/Projects/DWB.

HUND, E. naheliegend oder weiterhergeholt - unterrichtspraktische Überlegungen zu Raum und Körper in der Wissenschaftssprache. In: BARKOWSKI, H./WOLFF, A. (Hrsg.) Materialien Deutsch als Fremdsprache 52. Regensburg 1999, 290-320.

HuTz, M. Kontrastive Fachtextlinguistik für den fachbezogenen Fremdsprachenunterricht. Trier, Wissenschaftlicher Verlag 1997.

JoHnEN, T. Die Kennzeichnung von Handlungszielen durch Modalverben im Deutschen und Portugiesischen. In: GÄRTNER, E./ Heruth, M. J. Peres/ Sommer, N. (EDS.): Contribuições para a Didáctica do Português Língua Estrangeira: Akten der Sektion "Didaktik des Portugiesischen als Fremdsprache" des 4. Deutschen Lusitanistentages (Universität Mainz in Germersheim), 11. bis 14. September 2001. Frankfurt am Main, TFM 2003, 109-143.

KAISER, D. Wege zum wissenschaftlichen Schreiben. Eine kontrastive Untersuchung zu studentischen Texten aus Venezuela und Deutschland. Tübingen, Stauffenburg 2002.

MEURER, J. L.“Aspectos da textualização da introdução a artigos acadêmicos de pesquisadores brasileiros. In: Círculo de Estudos Lingüísticos do Sul, 1995, Florianópolis, Caderno de Resumos do CELSUL. Florianópolis, UFSC 1995, 18-18.

REDDER, A. /BRÜNNER, G. Studien zur Verwendung der Modalverben. Tübingen, Narr 1983.

REDDER, A. Modalverben in wissenschaftlicher Argumentation - Deutsch und Englisch im Vergleich. In: Jahrbuch Deutsch als Fremdsprache. München, iudicium 2001, 313-331.

ReHBEIN, J. Komplexes Handeln. Elemente zur Handlungstheorie der Sprache. Stuttgart, Metzler, 1977.

REUTER, E. Wissenschaftliches Schreiben im Umbruch. Über einige Folgen der Globalisierung in den Fremdsprachenphilologien. In: Info DaF 4/30. München, iudicium 2003.

Swales, J. Genre Analysis: English in Academic and Research Settings. Cambridge, Cambridge University Press, 1990.

Swales, J. Aspects of Article Introductions. Language Studies Unit, The University of Aston, Birmingham, 1981.

TAYLOR, G./CHEN, T. Linguistic, cultural, and subcultural issues in contrastive discourse analysis: Anglo-American and Chinese scientific texts. In: Applied Linguistics 12/1991, 317-336.

Widdowson, H. G. Explorations in Applied Linguistics. Oxford, Oxford University Press 1979.

Wunderlich, D. Modalverben im Diskurs und System. In: Rosengren, I. (Hrsg.) Sprache und Pragmatik. Lund, CWK Gleerup, 1981, 11-53. 


\section{Korpus:}

FISCHHABER, K. Digitale Ethnographie in der Sprach- und Kulturvermittlung. Magisterarbeit. München, LMU, 2004. [D24]

KöRNER, C. Partikelanalysen und Partikelvermittlung am Beispiel des Thailändischen und des Deutschen. Magisterarbeit. München, LMU, 2005. [D13]

KURZ GatTI, I. O modo subjuntivo em alemão e português. Magisterarbeit. São Paulo, USP, 2001. [B11]

PutTinger, N. Das Konzept der Interkulturalität in neueren Lehrwerken für Deutsch als Fremdsprache. Magisterarbeit. München, LMU, 1995. [D14]

MAIR, D. Konzept-Drehbuch für Internet-Lehrwerke im Bereich DaF - unter Berücksichtigung lernpsychologischer und didaktischer Aspekte multimedialer Anwendungen.

Magisterarbeit. München, LMU, 1999. [D23]

SCHEFFELMEIER, M. P. A tradução técnica no meio empresarial: Um estudo descritivo de Caso. Magisterarbeit. São Paulo, USP, 2001. [B3]

SCHWEIGER, K. Zur Vermittlung der Nominalplurale an DeutschlernerInnen Lehrwerkpräsentation und Lernerwahrnehmung. Magisterarbeit. München, LMU, 2005. [D5]

SEIXAS, A. P. DE. Estratégias de aprendizagem para a compreensão auditiva do alemão como língua estrangeira. Magisterarbeit. São Paulo, USP, 2003. [B10]

SHIRAI-ELFEKY. K. Haiku und Jahreszeiten - ein interkultureller Vergleich. Magisterarbeit. München, LMU, 2007. [D17]

SILVA, R. F. DA. A entoação em sentenças interrogativas do alemão e do português do Brasil. Magisterarbeit. São Paulo, USP, 2003. [B4] 